\title{
The influence of guar gum on intestinal cholesterol transport in the rat
}

\author{
BY JENNIFER M. GEE, N. A. BLACKBURN AND I. T. JOHNSON \\ ARC Food Research Institute, Colney Lane, Norwich NR4 7UA
}

(Received 11 January 1983 - Accepted 4 May 1983)

\begin{abstract}
1. Everted sacs of rat proximal small intestine were used to determine the effect of guar gum (5 g/l) on the uptake of cholesterol $(0 \cdot 1 \mathrm{mM})$ from a solution of micelles.

2. The uptake of cholesterol was found to be linear both in the presence and absence of guar gum. When guar was present throughout the whole of the incubation medium, the uptake of cholesterol was reduced to approximately $40 \%$ of control values. Sacs which had been pre-incubated in guar gum before exposure to cholesterol in a guar-free medium also showed a reduction in cholesterol uptake but this was less pronounced.

3. A two-stage perfusion technique, previously described (Blackburn \& Johnson, 1981), was used to determine the effect of a guar layer adsorbed to the mucosal surface on cholesterol absorption in vivo. Such a layer leads to a reduction of approximately $36 \%$; it was concluded that guar slows the absorption of cholesterol from micelles by a mechanism, or mechanisms, involving an increased resistance to diffusion in the aqueous medium.

4. Groups of rats were meal-fed for at least $30 \mathrm{~d}$ on semi-synthetic diets with or without the inclusion of guar gum $(20 \mathrm{~g} / \mathrm{kg})$. Rates of intestinal absorption of cholesterol, glucose and fluid were then determined by the perfusion technique in vivo. There was no reduction in absorption in the test animals compared with the controls.

5. It is proposed that guar gum is able to slow the intestinal transport of cholesterol from a suspension of pre-formed micelles, but only when both are present in the lumen together. No evidence was obtained to suggest that the consumption by rats of a diet containing guar gum, at a level similar to that used in human studies, leads to any adaptive reduction in their rates of cholesterol or glucose absorption.
\end{abstract}

There is much current interest in the beneficial effects of certain types of dietary fibre, which have been shown to improve glucose tolerance and reduce plasma cholesterol levels in man (Anderson \& Chen, 1979). In particular, two recent studies have suggested that guar gum, a water-soluble storage polysaccharide derived from the cluster bean (Cyamopsis tetragonoloba), may be of therapeutic value in the treatment of hypercholesterolaemia (Jenkins et al. 1980; Khan et al. 1981). The mechanisms underlying the hypocholesterolaemic effect of guar gum are not understood, but one commonly proposed possibility is that it may interfere with the intestinal absorption of cholesterol. The purpose of the present study was to observe the effect of guar gum on the uptake of cholesterol from pre-formed micelle solutions under controlled experimental conditions. A further aim was to explore the possibility that the long-term consumption of guar gum, at a level comparable to that which has proven effective in man, might lead to adaptive changes in intestinal cholesterol absorption in the rat.

\section{MATERIALS AND METHODS \\ Cholesterol transport in vitro}

Male Wistar rats (200-300 g) were allowed food (Labsure CRM, Christopher Hill Group Ltd, Poole, Dorset) and water ad lib. before killing by stunning and decapitation, and the preparation of everted sacs $(30-40 \mathrm{~mm})$ of proximal small intestine $(150-500 \mathrm{~mm}$ from the pyloric sphincter). All incubation media were prepared from Krebs-bicarbonate buffer, modified by the omission of calcium in the case of the medium containing micelles. The final micelle suspension contained (mM) sodium taurocholate 5 , monolein $0 \cdot 3$, oleic acid $0 \cdot 6$, cholesterol $0 \cdot 1$, together with $\left[{ }^{3} \mathrm{H}\right]$ cholesterol $(91 \mu \mathrm{Ci} / 1)$ and ${ }^{14} \mathrm{C}$-labelled polyethylene glycol (43 $\mu \mathrm{Ci} / \mathrm{l} ; \mathrm{PEG})$ (Amersham International, Amersham, Bucks). A clear suspension of micelles was obtained after sonication (Chow \& Hollander, 1978). Glucose (10 mM) was 
also included unless otherwise indicated. Where appropriate, guar gum was dispersed in the media by the addition of the dry powder (Gum Guar; Sigma, Poole, Dorset) during high-speed vortex mixing (Ultra-Turrax; Janke and Kunkel KG, Staufen i. Br. FDR). Such media were then allowed to stand for at least $2 \mathrm{~h}$ before use to allow the full development of viscosity, as determined with a rotary viscometer at a continuous shear rate of $50 / \mathrm{s}$ (Epprecht Rheomat 15; Contraves AG; Zurich, Switzerland). Sacs were incubated in $25 \mathrm{ml}$ Erlenmeyer flasks containing $10 \mathrm{ml}$ medium gassed continuously with oxygen-carbon dioxide $(95: 5 \mathrm{v} / \mathrm{v})$ shaken at 120 oscillations $/ \mathrm{min}$ and maintained at $37^{\circ}$. At timed intervals the sacs were removed and drained briefly by touching the lower end on dry filter paper. The serosal contents and the ligatured ends of each sac were discarded and the remaining tissue was placed in glass vials and dried to constant weight overnight. The dried residues were dissolved in concentrated nitric acid $(0.4 \mathrm{ml})$ and mixed with $0.75 \mathrm{M}$-Trizma base $(3.6 \mathrm{ml})$; portions $(0.5 \mathrm{ml})$ were diluted to $1 \mathrm{ml}$ with distilled water and added to $9 \mathrm{ml}$ scintillation fluid (Cocktail T Scintran; BDH, Poole, Dorset).

The ${ }^{3} \mathrm{H}$ and ${ }^{14} \mathrm{C}$ activities of such samples were determined by dual-label counting on a Philips PW4700 liquid-scintillation counter, with automatic quench compensation; counting efficiency was measured by external standard channels ratio.

\section{Cholesterol and glucose transport in vivo}

The absorption of cholesterol, glucose and water was determined in vivo by means of an isolated-loop technique in anaesthetized rats. After the establishment of anaesthesia by an intraperitoneal injection of sodium pentobarbitone (Sagatal; May and Baker Ltd; Dagenham, Essex), the small intestine was exposed by a mid-line ventral incision. The jejunum (approximately $100-500 \mathrm{~mm}$ from the pyloric sphincter) was isolated and glass cannulas (internal diameter $2 \mathrm{~mm}$ ) were tied in place at either end. After flushing with Ringer solution, the isolated segment was divided into two lengths by the insertion of two further cannulas at the mid-point. The intestine was then returned to the abdominal cavity, the inlet cannulas were connected to a multi-channel pump and a flow (approximately $1 \mathrm{ml} / \mathrm{min}$ ) established in an aboral direction. Exact flow rates were determined gravimetrically. Collections (2-4 min) of effluent were made from each segment directly into pre-weighed polythene scintillation vials. At the end of the experiment the animal was killed, the segments were drained, removed and dried to constant weight at $85^{\circ}$. Each perfusate sample was weighed, mixed with scintillant and assayed for ${ }^{3} \mathrm{H}$ and ${ }^{14} \mathrm{C}$ content as described. All perfusions were performed under an infra-red lamp (ambient temperature approximately $37^{\circ}$ ) with the abdomen protected with moistened gauze.

Rates of absorption of cholesterol, glucose and fluid were calculated using the simple relationship: input $/ \mathrm{min}$ - output $/ \mathrm{min}=$ absorption $/ \mathrm{min}$, the initial substrate concentration and the perfusion pump rate having been accurately determined. The validity of the calculation depends on the volume of the perfused loops remaining constant during the course of the experiment. This was checked by recalculating the results for the last $20 \mathrm{~min}$ of each perfusion using the concentration of non-absorbable marker ${ }^{14} \mathrm{C}$-labelled inulin as an indicator of fluid absorption. Transport was approximately linear over this period. For each experiment, the lines of best fit for the experimental points obtained by the two methods were estimated by the method of least squares and within each group the slopes of such lines were compared by Student's paired $t$ test. The slopes of the lines obtained by the two methods did not differ significantly.

To investigate the direct effect of guar gum on cholesterol absorption, the two stage perfusion procedure described elsewhere was employed (Blackburn \& Johnson, 1981) using male Wistar rats (200-300 g) allowed food and water ad lib. The first-stage perfusate in the control loop consisted of guar-free Ringer solution, whilst that in the test loop contained 
Table 1. Composition of diets $(\mathrm{g} / \mathrm{kg}$ diet $)$

\begin{tabular}{lcc}
\hline \hline Ingredients & Control diet & Guar diet \\
\hline Starch* & 290 & 270 \\
Sucrose & 290 & 290 \\
Casein $\dagger$ & 200 & 200 \\
Maize oil & 80 & 80 \\
Cellulose $\$$ & 80 & 80 \\
Mineral mix & 40 & 40 \\
Vitamin mix $\$$ & 20 & 20 \\
Guar gum & - & 20 \\
\hline
\end{tabular}

* 'Snoflake' maize flour; Corn Products Ltd, Manchester.

$\dagger$ Edible casein; Glaxo Farley Foods, Plymouth.

* Solka-floc; Johnson, Jörgensen, Wettre Ltd, London.

Produced the following levels of minerals in the diet (g/kg diet): $\mathrm{CaHPO}_{4} 13, \mathrm{CaCO}_{3} 8 \cdot 2, \mathrm{KCl} 7 \cdot 03, \mathrm{Na}_{2} \mathrm{HPO}_{4}$ $7 \cdot 4, \mathrm{MgSO}_{4} \cdot \mathrm{H}_{2} \mathrm{O} 4 \cdot 0, \mathrm{MnSO}_{4} . \mathrm{H}_{2} \mathrm{O} 0 \cdot 18, \mathrm{ZnCO}_{3} 0 \cdot 03, \mathrm{FeSO}_{4} .7 \mathrm{H}_{2} \mathrm{O} 0 \cdot 144, \mathrm{CuSO}_{4} 0 \cdot 015, \mathrm{KIO}_{3} 0 \cdot 001$.

$\S$ Contained all necessary vitamins at levels equal to, or above, those recommended by the (US) National Research Council (1972) for growth and reproduction.

I Sigma Chemical Co., St Louis, MO.

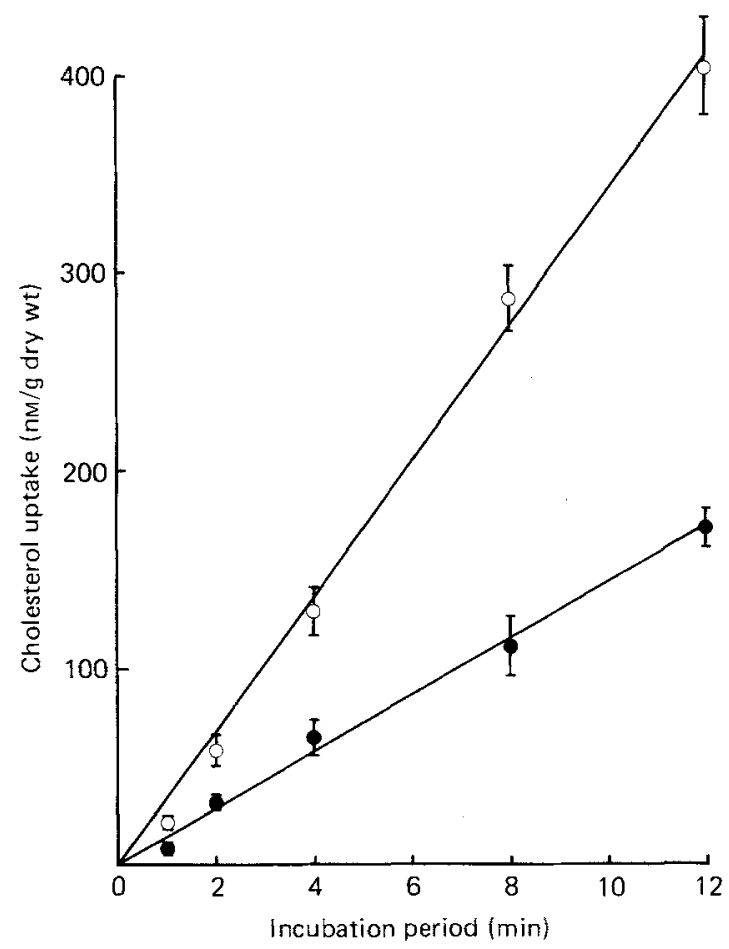

Fig. 1. Uptake of cholesterol (nM/g dry weight) $v$. period of incubation (min) incubated with $(\mathbf{C})$ or without $(O)$ guar gum. Five test and five control sacs were prepared from each animal. Points are mean values with their standard errors represented by vertical bars, for single sacs from eight animals. All differences were statistically significant $(P<0 \cdot 05)$. 


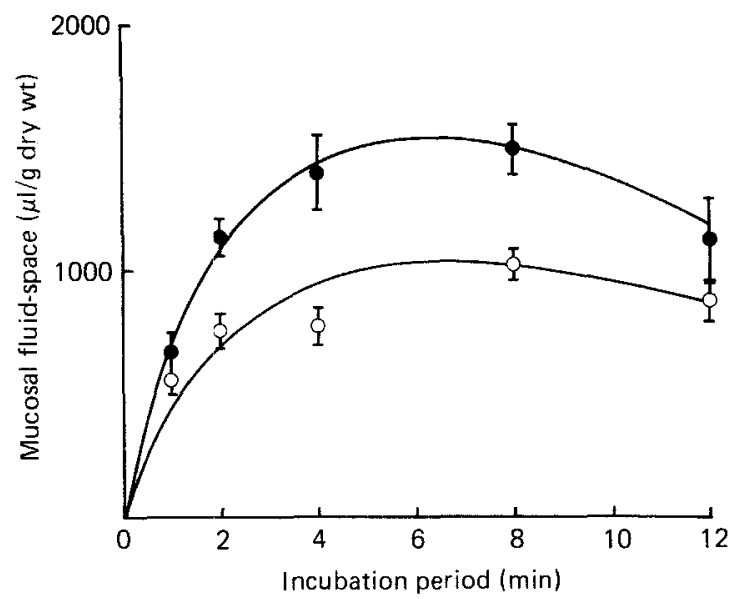

Fig. 2. Mucosal fluid-space of everted rat intestine, calculated from the apparent uptake of ${ }^{14} \mathrm{C}$-labelled polyethylene glycol by the sacs in Fig. 1. Points are mean values with their standard errors represented by vertical bars. All differences after $1 \mathrm{~min}$ were statistically significant $(P<0.02)$.

guar gum $(6 \mathrm{~g} / 1)$. In the second stage, the guar-free perfusate in both loops consisted of pre-formed micelles in Ringer solution as described, labelled with $\left[{ }^{3} \mathrm{H}\right]$ cholesterol $(400 \mu \mathrm{Ci} / 1)$ and ${ }^{14} \mathrm{C}$-labelled inulin $(18 \mu \mathrm{Ci} / 1$; Amersham International).

For the guar-feeding study, thirty male Wistar rats (initial weight 125-150 g) were housed in groups of four in wire-bottomed cages and trained to meal-feed (09.00-10.00 hours) on either the control or the test semi-synthetic diet (Table 1), water being allowed ad lib. Animals were maintained on this regimen for at least $30 \mathrm{~d}$ before the perfusion studies; no significant differences in food consumption or final weights were observed. Animals from the test and control groups were operated on $4 \mathrm{~h}$ (fed animals) or approximately $26 \mathrm{~h}$ (fasted animals) after the final meal. The object of this was to expose any inhibitory effect due to the persistence of guar gum in the intestine. One loop in each animal was perfused with the solution containing labelled micelles, the other with Ringer solution containing glucose (10 mM) labelled with $\left[{ }^{3} \mathrm{H}\right]$ glucose $(35 \mu \mathrm{Ci} / 1$; Amersham International); both solutions also contained labelled inulin as described. Cholesterol and glucose uptake were measured in the appropriate loops, whilst fluid uptake was determined simultaneously in both. The uptake of all three was found to be close to linearity after the first 20 min of each perfusion; rates were therefore estimated by linear regression for each experiment over the period $t=20$ to $t=50 \mathrm{~min}$.

\section{ST A TISTICS}

The significance of differences between means was estimated by Student's unpaired $t$ test when comparisons were made between animals, as in the guar-feeding study. In experiments where comparisons were made between loops or everted sacs from the same animal, Student's paired $t$ test was used.

\section{RESULTS}

Effect of guar gum on cholesterol uptake in vitro

The uptake of cholesterol by everted sacs, in the presence or absence of guar gum $(0.5 \%$; $\left.0.098 \mathrm{Ns} / \mathrm{m}^{2}(98 \mathrm{cP})\right)$ is illustrated in Fig. 1. In both cases the uptake of cholesterol was linear with time over the $12 \mathrm{~min}$ incubation period, but the presence of guar gum brought about a reduction of approximately $60 \%$ in the rate of uptake compared with control sacs. In order to determine the uptake of cholesterol by the mucosal cells accurately, it was 


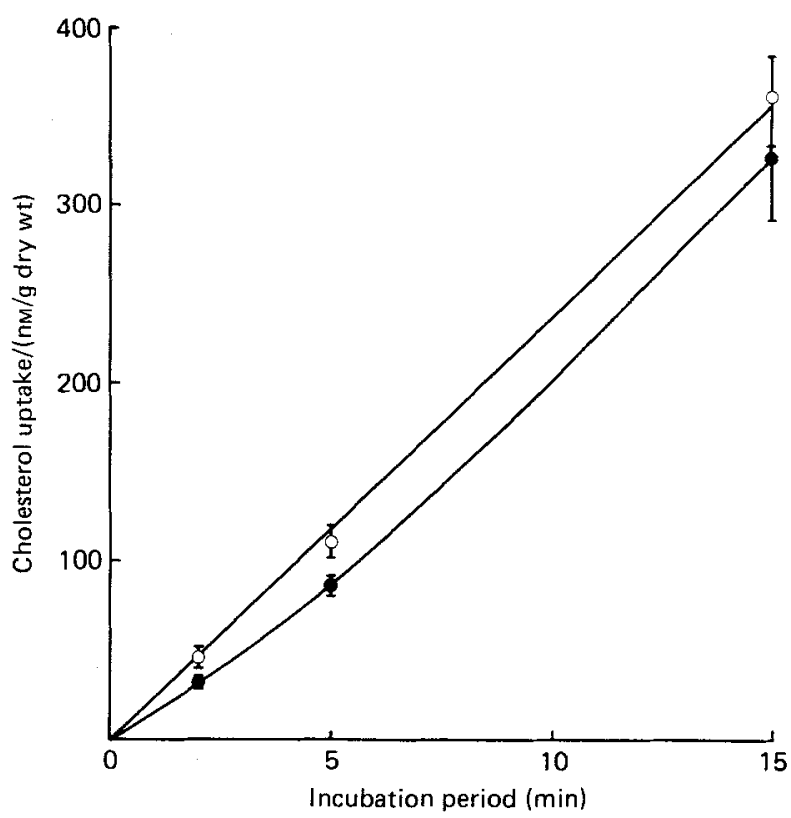

Fig. 3. Uptake of cholesterol (nM/g dry weight) $v$. period of incubation ( $\mathrm{min}$ ) by everted sacs of rat intestine pre-incubated in buffer alone $(O)$ or in buffer containing guar gum $(O)$. Points are mean values with their standard errors represented by vertical bars, for single sacs from eight animals. Differences at $2 \mathrm{~min}$ and $5 \mathrm{~min}$ were statistically significant $(P<0.01)$.

necessary to correct for the label present in the fluid layer associated with the mucosal surface, the volume of which was calculated from the adhering non-absorbable marker, PEG. It is noteworthy that both the volume and the time required for the mucosal fluidspace to reach equilibrium with the incubation medium were increased by the presence of guar gum. This is illustrated in Fig. 2 which shows the apparent volume of the mucosal fluid-space at each of the sampling times.

The increased mucosal fluid-space in the sacs incubated with guar gum indicates the presence of a viscous fluid layer entrapped by the mucosa when the sac is removed from the incubation medium. As we have shown elsewhere, the presence of this guar layer leads to an increased resistance to diffusion to the mucosal surface and a reduction in glucose uptake in such sacs when subsequently incubated in a guar-free medium (Johnson \& Gee, 1981). The effects of pre-incubation in guar gum on cholesterol uptake during a subsequent incubation are illustrated in Fig. 3. Six everted sacs were prepared from each rat and incubated for $15 \mathrm{~min}$ with (three test sacs) or without (three control sacs) guar gum (0.5\%; $0.098 \mathrm{Ns} / \mathrm{m}^{2}$ ). All sacs were then rinsed for $1 \mathrm{~min}$ and transferred to a glucose-free incubation medium containing micelles. Paired test and control sacs were removed after the intervals indicated. The uptake of cholesterol by the test sacs was significantly lower than that of the controls $(P<0.01$; assessed by Student's paired $t$ test $)$ after incubation periods of 2 and $5 \mathrm{~min}$. Thereafter the rates of uptake appeared to be approximately equal and no significant difference was observed between test and control sacs after $15 \mathrm{~min}$ incubation.

\section{Effect of guar gum on cholesterol uptake in vivo}

The principle of the two-stage incubation regimen applied to everted sacs was extended to a more intact intestinal preparation in vivo using a procedure similar to that described by Blackburn \& Johnson (1981). Test and control loops were perfused with or without guar 


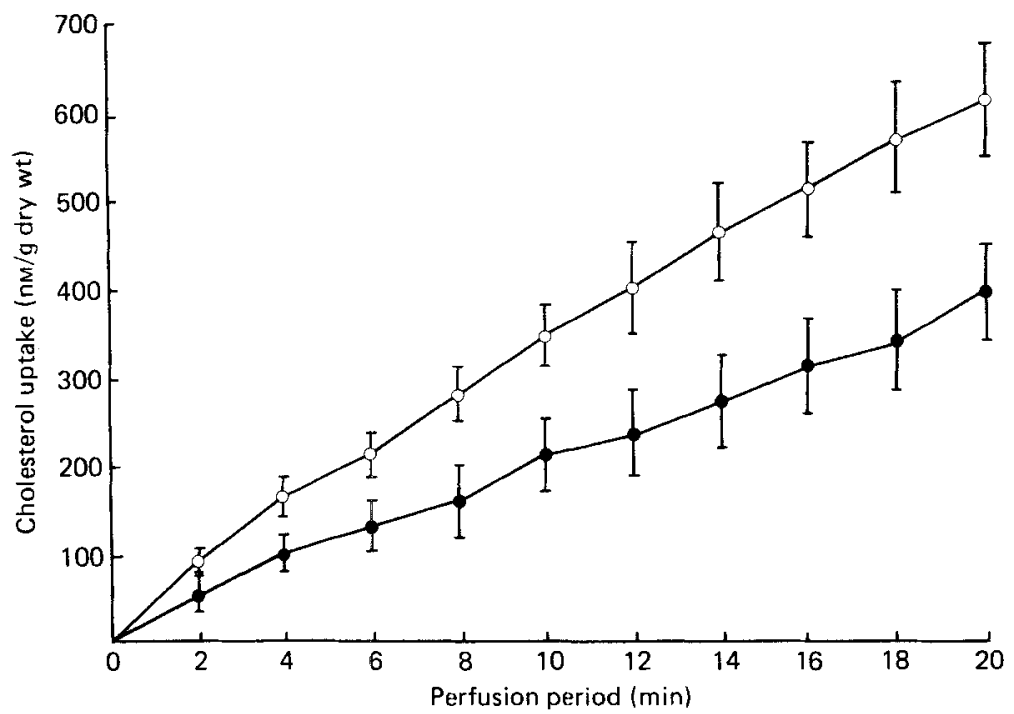

Fig. 4. Uptake of cholesterol (nM/g dry weight) $v$. period of perfusion ( $\mathrm{min}$ ) by loops of rat intestine, pre-perfused with Ringer solution only $(O)$ or with Ringer solution containing guar gum $(\bullet)$. Points are mean values with their standard errors represented by vertical bars for eight animals.

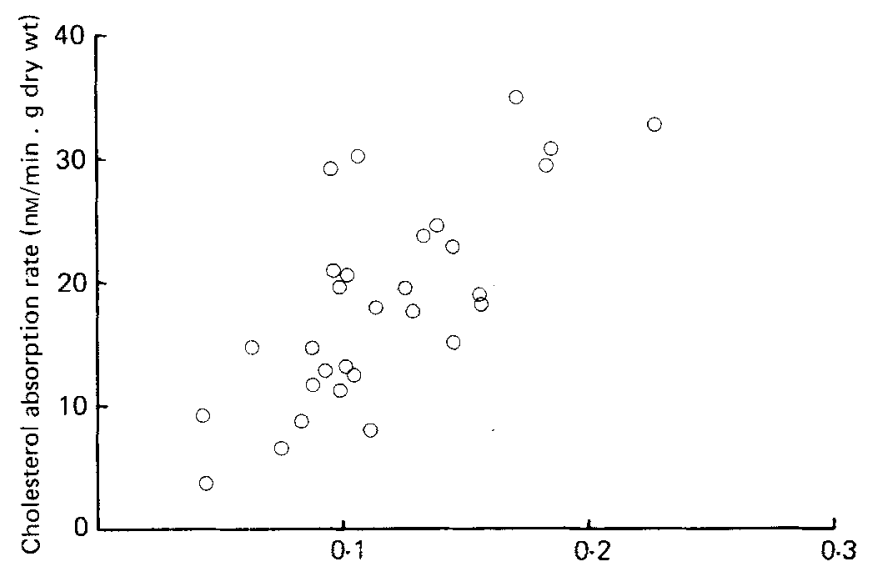

Fluid absorption rate $(\mathrm{ml} / \mathrm{min}$. g dry wt $)$

Fig. 5. Scatter diagram relating the rate of cholesterol absorption (nM/min per $\mathrm{g}$ dry weight) to that of fluid absorption ( $\mathrm{ml} / \mathrm{min}$ per $\mathrm{g}$ dry weight of tissue) for the thirty experiments summarized in Table 2 $(r 0.730, P<0.001)$.

gum $(6 \mathrm{~g} / \mathrm{l})$ and the uptake of cholesterol during a subsequent guar-free perfusion was determined. As illustrated in Fig. 4, cholesterol uptake was approximately linear with time, though the line for the control loops showed some signs of curvature over the full course of the experiment. The rate of uptake by the test loops was reduced by approximately $36 \%$ relative to the controls.

\section{Guar-feeding study}

Average rates of cholesterol and glucose uptake, together with fluid transport, in the fed or fasted guar and control groups are summarized in Table 2. Cholesterol uptake was not significantly affected by fasting in the guar-fed group and neither value was significantly 
Table 2. Rates of absorption of cholesterol, glucose and fluid from loops of rat jejunum, perfused in vivo

(Mean values with their standard errors; no. of animals in parentheses)

\begin{tabular}{|c|c|c|c|c|c|c|c|c|}
\hline & \multicolumn{2}{|c|}{$\begin{array}{c}\text { Cholesterol } \\
(\mathrm{nmol} / \mathrm{min} \text { per } \mathrm{g})\end{array}$} & \multicolumn{2}{|c|}{$\begin{array}{c}\text { Fluid } \\
(\mathrm{ml} / \mathrm{min} \text { per } \mathrm{g})\end{array}$} & \multicolumn{2}{|c|}{$\begin{array}{c}\text { Glucose } \\
(\mu \mathrm{mol} / \mathrm{min} \text { per } \mathrm{g})\end{array}$} & \multicolumn{2}{|c|}{$\begin{array}{c}\text { Fluid } \\
(\mathrm{ml} / \mathrm{min} \text { per } \mathrm{g})\end{array}$} \\
\hline & Mean & SE & Mean & SE & Mean & $\mathrm{SE}$ & Mean & $\mathrm{SE}$ \\
\hline \multicolumn{9}{|l|}{ Guar group } \\
\hline Fed $(7)$ & $20 \cdot 7^{*}$ & $3 \cdot 2$ & $0.14^{*}$ & 0.02 & $4 \cdot 56$ & 0.33 & $0 \cdot 16$ & 0.01 \\
\hline Fasted (7) & 22.4 & $3 \cdot 2$ & 0.14 & 0.01 & $5 \cdot 16$ & $0 \cdot 34$ & $0 \cdot 13$ & 0.02 \\
\hline $\begin{array}{l}\text { Statistical significance } \\
\text { of difference: } P \dagger\end{array}$ & NS & - & NS & - & NS & - & NS & - \\
\hline \multicolumn{9}{|l|}{ Control group } \\
\hline Fed $(8)$ & $11 \cdot 4$ & $1 \cdot 7$ & 0.09 & 0.01 & 3.94 & $0 \cdot 24$ & 0.14 & 0.01 \\
\hline Fasted (8) & $20 \cdot 0$ & $2 \cdot 5$ & $0 \cdot 10$ & 0.01 & 5.49 & 0.50 & 0.13 & 0.02 \\
\hline $\begin{array}{l}\text { Statistical significance } \\
\text { of difference: } P^{\dagger}\end{array}$ & 0.02 & - & NS & - & 0.02 & - & NS & - \\
\hline
\end{tabular}

NS, not significant.

Mean values for fed animals in guar group were significantly different from those in the control group:

* $P<0.05$.

$\dagger$ Indicates significance of differences between mean values for fed or fasted animals within the guar-fed or control groups.

different from the fasted control group. However, the fed animals in the control group showed significantly slower cholesterol uptake than those in the guar group. No impairment of glucose transport was observed in the guar-fed rats.

There was a tendency for the fluid uptake in the loops perfused with glucose to be higher than in the glucose-free loops perfused with cholesterol. This is to be expected, as it is well established that glucose stimulates fluid uptake in the small intestine (Parsons \& Wingate, 1961).

Fig. 5 is a scatter diagram relating the rates of fluid and cholesterol absorption for the thirty animals used in this study; the correlation coefficient for the values was significantly different from zero $(r 0.73 ; P<0.001)$.

\section{DISCUSSION}

In the course of its absorption, dietary cholesterol must first be incorporated into micelles in the gut lumen and then repartitioned into the aqueous phase immediately adjacent to the absorptive surfaces of the villi. Under experimental conditions, both the transfer of micelles through the poorly stirred mucosal fluid layer and the subsequent passive uptake of free cholesterol by the brush-border membrane have been shown to be potentially significant as rate-limiting steps for the over-all transport process (Chow \& Hollander, 1978; Thomson, 1980). The present studies indicate that the uptake of cholesterol from pre-formed micelles is markedly susceptible to the presence of guar gum in the incubation medium; several possible mechanisms might account for this.

In previous reports we have shown that viscous polysaccharides such as guar gum slow the intestinal transport of sugars, an effect we ascribe to an increased resistance to the diffusion of substrates in the mucosal fluid layer (Blackburn \& Johnson, 1981; Johnson \& Gee, 1981). Similarly, the inhibitory effect of guar gum on the uptake of cholesterol might simply be due to a reduction of turbulence and stirring and, hence, of convective solute movement in the fluid layer overlying the villi. An alternative possibility is that guar gum 
may interfere directly with cholesterol transport, perhaps by restricting the diffusion of the relatively large and bulky micelles, or by chemically binding cholesterol or other micelle constituents, and removing them from solution altogether (Vahouny et al. 1980 b). The results of the present study suggest that both these mechanisms may be operative. Under the simple conditions of the one-stage everted-sac procedure, guar gum was present in the bulk-phase of the incubation medium and brought about a marked reduction in cholesterol transport which, nevertheless, remained linear with time. During the two-stage incubation with everted sacs, and the two-stage perfusion procedure, guar gum was present only as a thin layer adsorbed on to the mucosal surface. Under these conditions, although the concentration of polysaccharide is low, its presence leads to an increase in the apparent thickness of the mucosal unstirred layer (Johnson \& Gee, 1981). In view of the low levels of guar gum present under these conditions, it seems unlikely that cholesterol binding is the primary mechanism leading to reduced uptake, unless the binding sites are of very high affinity. It is noteworthy that in the case of the everted sacs, the effect of the adsorbed layer was temporary and less than that of guar gum dispersed uniformly throughout the incubation medium. This suggests that both the surface layer effect and a direct binding of cholesterol by guar gum in the bulk of the medium together contribute to the over-all restriction of cholesterol uptake.

Apart from the direct influence on nutrient absorption exerted by fibre actually present in the lumen, it has recently been suggested that the prolonged consumption of some components of dietary fibre may lead to adaptive changes in the intestinal mucosa, giving rise to a reduced physiological capacity for active and passive nutrient absorption (Vahouny et al. 1978, 1980a; Schwartz \& Levine, 1980). The aim of the guar-feeding study was to look for such an effect, following the long-term consumption of a dietary supplement of guar gum by rats. The supplement was superimposed on a diet which already contained fibre in the form of cellulose and the level was chosen to correspond, so far as such comparisons are possible, to that which might be said to be of nutritional or clinical significance in man. A guar gum supplement of $20 \mathrm{~g} / \mathrm{kg}$ would correspond to a daily intake of $10 \mathrm{~g}$ in man, assuming an average consumption of $500 \mathrm{~g}$ dry food $/ \mathrm{d}$. Similar levels have been shown to be acceptable to human subjects in extended clinical trials and are associated with reduced levels of blood cholesterol (Jenkins et al. 1980; Khan et al. 1981). Our experimental regimen was designed to test both for a physiological adaptation of the mucosa and for the possible persistence of a layer of guar gum within the gut lumen in the guar-supplemented rats. Such a layer might conceivably interfere with transport during a subsequent perfusion.

As we have shown previously (Blackburn \& Johnson, 1981), animals consuming guar gum at the level employed in the present study have characteristically viscous stomach contents which are resistant to wetting and dispersal. The small intestinal chyme in such animals also has a high apparent viscosity, exceeding that which gave rise to reduced rates of glucose absorption in vivo. As might be expected, the emptying of food from the stomachs of such rats is slowed relative to control animals, and there are significant increases in the length and transmural thickness of the small intestine (N. A. Blackburn and I. T. Johnson, unpublished results). Despite these indications of modified intestinal functioning and growth, no adverse effects were observed in the guar-supplemented animals and no reduction occurred in their growth rates or food consumption.

No evidence was obtained in the present study for any adaptive reduction in cholesterol or hexose transport. Nor, it should be noted, was any evidence obtained for our earlier suggestion that such an effect might be mimicked by the persistence of non-digestible polysaccharides in the gut during the post-prandial period (Blackburn et al. 1982). Our findings do not conform to the general conclusions of other workers that prolonged 
consumption of various components of dietary fibre leads to adaptive reductions in cholesterol absorption (Vahouny et al. 1978, 1980a) or glucose absorption (Schwartz \& Levine, 1980). There are, of course, major differences between our study and those of previous workers; the most important perhaps is that we have included cellulose in both the control and test diets. Under normal circumstances no human diet, still less any rat diet, is completely fibre-free, and such a diet may itself alter the functional state of the mucosa. It is known, for example, that an elemental diet leads to a reduction in mucosal cell turnover and villus height in rat small intestine (Ecknauer et al. 1981). We suggest therefore that the approach used here, in which a specific component of dietary fibre is added to a diet containing a basal level of fibre in the form of cellulose, has greater physiological validity, given that the intention is to use the animal model to assess the probable effect of particular materials in human diets.

The positive correlation between rates of cholesterol and water absorption observed by us emphasizes the importance of unstirred layer effects in determining rates of cholesterol uptake in the intestine. The probable explanation is that a net flux of water from the lumen of the intestine toward the mucosal surface tends to wash micelles into the unstirred water layer and leads to an increase in the over-all effective permeability of the mucosa to cholesterol (Miyamoto et al. 1982). This suggests that in the intact animal, factors which give rise to changes in fluid secretion and absorption in the gastrointestinal tract might modify the absorption of cholesterol and other passively transported nutrients as a secondary effect.

We conclude that when present in the fluid phase overlying the mucosa, guar gum brings about a marked inhibition of intestinal cholesterol uptake. The presence of guar gum in the partially digested contents of the small intestine is likely, therefore, to lead to a reduction in the rate of cholesterol uptake in man. Since the absorption of cholesterol from the human diet is limited (Wilson et al. 1968) such an effect could well lead to increased faecal excretion of dietary cholesterol and play at least a contributary role in the hypocholesterolaemic action of guar gum supplements.

The mechanism of action of guar seems to be similar to that already described by us in relation to actively absorbed sugars (Blackburn \& Johnson, 1981; Johnson \& Gee, 1981), although the importance of physical interactions between guar gum and micelles needs further study and the secondary effect of reduced fluid movement in the presence of guar gum may also be important. The absence of any evidence for adaptive changes in the absorptive capacity of the small intestines of the guar-supplemented rats used in this study suggests that such effects are unlikely to be important at dietary levels which have practical significance for man.

The authors wish to acknowledge the technical assistance of Ms J. Brown and Ms V. Simmonds during the course of this work.

\section{REFERENCES}

Anderson, J. W. \& Chen, W. (1979). American Journal of Clinical Nutrition 32, 346-363.

Blackburn, N. A. \& Johnson, I. T. (1981). British Journal of Nutrition 46, 239-246.

Blackburn, N. A., Johnson, I. T. \& Gee, J. M. (1982), Gastroenterology 82, 821.

Chow, S. L. \& Hollander, D. (1978). Lipids 13, 239-245.

Ecknauer, R., Sircar, B. \& Johnson, L. R. (1981). Gastroenterology 81, 781-786.

Jenkins, D. J. A., Reynolds, D., Slavin, B., Leeds, A. R., Jenkins, A. L. \& Jepson, E. M. (1980). American Journal of Clinical Nutrition 33, 575-581.

Johnson, I. T. \& Gee, J. M. (1981). Gut 22, 398-403.

Khan, A. R., Ghazala, Y. K., Mitchel, A. \& Qadeer, M. A. (1981). American Journal of Clinical Nutrition 34, 2446-2449

Miyamoto, Y., Hanano, M., Iga, T. \& Ishikawa, M. (1982). Journal of Pharmacobio-Dynamics 5, $445-447$. 
National Research Council (1972). Nutrient Requirements of Laboratory Animals, 2nd ed. p. 64. Washington DC: National Academy of Sciences.

Parsons, D. S. \& Wingate, D. L. (1961). Biochimica et Biophysica Acta 46, 170-183.

Schwartz, S. E. \& Levine, G. D. (1980). Gastroenterology 79, 833-836.

Thomson, A. B. R. (1980). Journal of Lipid Research 21, 687-698.

Vahouny, G. V., Roy, T., Gallo, L. L., Story, J. A., Kritchevsky, D. \& Cassidy, M. (1980a). American Journal of Clinical Nutrition 33, 2182-2191.

Vahouny, G. V., Roy, T., Gallo, L. L., Story, J. A., Kritchevsky, D., Cassidy, M., Grund, B. M. \& Treadwell, C. R. (1978). American Journal of Clinical Nutrition 31, S208-\$212.

Vahouny, G. V., Tombes, R., Cassidy, M. M., Kritchevsky, D. \& Gallo, L. L. (1980b). Lipids 15, $1012-1018$.

Wilson, J. D., Lindsey, C. A. \& Dietschy, J. M. (1968). Annals of the New York Academy of Sciences 149, 808-821. 